\title{
Pediatric Interventional Cardiology: A Specialty Comes of Age
}

\author{
ROBERT H. BEEKMAN, III, M.D. \\ From the Division of Pediatric Cardialogy, C.S. Mott Children's Hospital, University of Michigan, Ann Arhor, Michigan
}

\section{Introduction}

Pediatric interventional cardiology was conceived in the 1960 s by Dr. William Rashkind, emerged as a distinct subspecialty during the 1980s, and has come of age in the 1990s. At C.S. Mott Children's Hospital, the number of interventional pediatric cardiac catheterizations has grown rapidly during the past decade (Fig. 1). Therapeutic procedures currently comprise $35 \%-40 \%$ of all cardiac catheterizations performed at our center. Interventional cardiac catheterization offers a wide array of catheter-based procedures to treat congenital or acquired cardiovascular disease in patients from the newborn period to adulthood (Table 1). Pcrcutaneous halloon dilation is the treatment of choice for congenital pulmonary or aortic valve stenosis, and provides effective therapy for many children with pulmonary artery stenosis or coarctation of the aorta (native or recurrent). In addition to dilation procedures, implantable devices are available to treat a variety of congenital or acquired cardiovascular anomalies. Balloon-expandable stents have improved our capacity to care for many children with pulmonary artery stenosis or hypoplasia. Embolization coils are used to occlude the small-to-moderate patent ductus arteriosus, aortopulmonary collateral vessels, and surgical shunts. Umbrella type devices continue to undergo clinical investigation to evaluate their potential to treat secundum atrial septal defects, muscular ventricular septal defects, and the patent ductus arteriosus. Of course, radiofrequency ablation has revolutionized the treatment of children with arrhythmias supported by accessory pathways or ectopic foci. This

Address for reprints: Robert H. Beekman, III, M.D., Division of Pediatric Cardiology, C.S. Mott Children's I Iospital, MCHC F1310, Box 0204, 1500 E. Medical Center Drive, Ann Arbor, MI 48109. Fax: (313) 936-9470. special issue of the Journal of Interventional Cardiology reviews the current "state of the art" of pediatric interventional cardiology. Articles are presented by established authorities and, perhaps more important, by a number of young pediatric cardiologists who will help to lead this dynamic specialty into the next century.

As a maturing field, pediatric interventional cardiology faces new responsibilities that come with age: selfassessment and self-control. Self-assessment requires formal outcome studies (to define the risks and benefits of the procedures utilized in our specialty); and self-control demands development of training and practice guidelines (to assure that physicians performing interventional procedures are qualified to do so).

\section{Outcome Studies}

The acute effectiveness of most of the transcatheter interventions listed in Table 1 have already been evaluated. The field of pediatric interventional cardiology must now rigorously study longer term outcomes. What precisely are the late results and complications, and which patients are most likely to benefit from these novel interventional proccdures? Formal comparisons to older and more established surgical therapies are essential. Finally, outcome studies must also evaluate the cost-effectiveness of transcatheter therapies. In his review article entitled "Cost-Effectiveness Analysis of Interventions for Congenital Heart Disease," Dr. Darryl Gray makes a plea for formal costeffectiveness studies in pediatric interventional cardiology. As recently demonstrated, the findings of a formal cost-effectiveness study may not necessarily support the preconceived notions of some interventional cardiologists. ${ }^{\prime}$ 


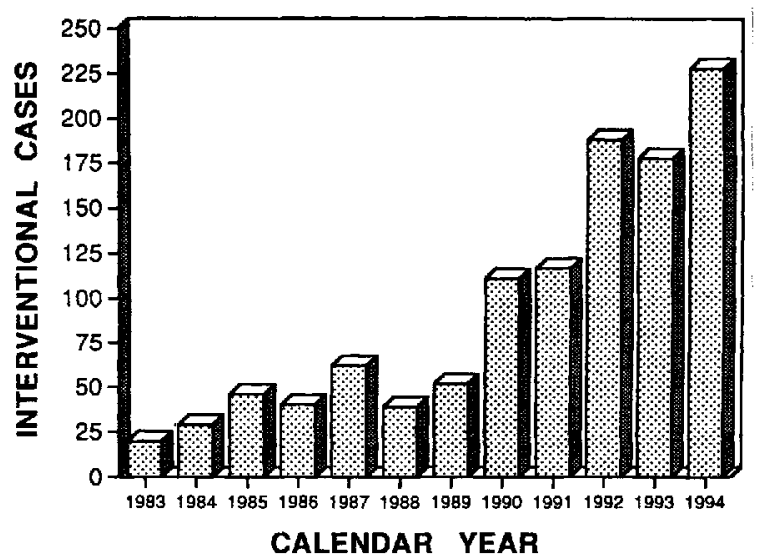

Figure 1. Interventional cases from 1983 to 1994.

Table 1. Therapeutic Transcatheter Techniques in Pediatric Cardiology, 1995

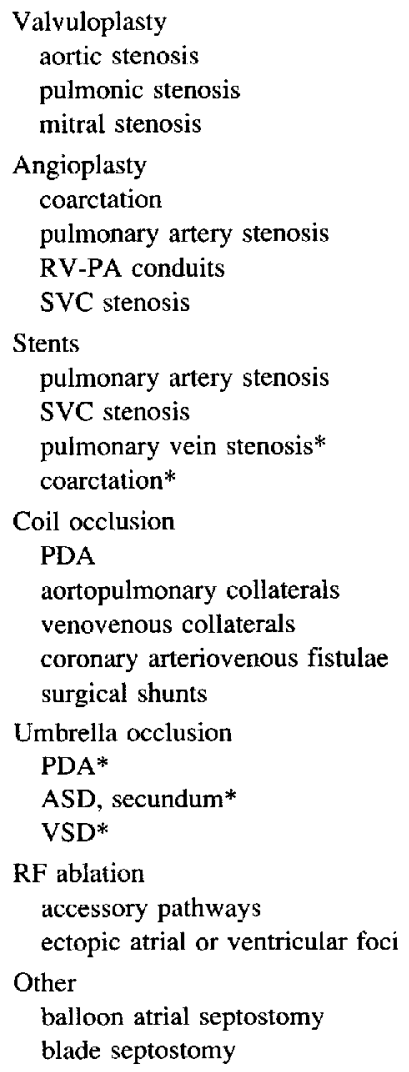

ASD $=$ atrial septal defect: PDA $=$ patent ductus arteriosus $\mathrm{RF}=$ radiofrequency; $\mathrm{RV}-\mathrm{PA}=$ right ventricular to pulmonary artery; SVC $=$ superior vena cava; $*$ investigational.

\section{Training and Practice Guidelines}

It is becoming clear that not all pediatric cardiologists can or should perform therapeutic transcatheter procedures. ${ }^{2}$ Nevertheless, formal training and practice guidelines are lacking in our specialty. In contrast, formal guidelines have been recognized in medical (adult) interventional cardiology and cardiac catheterization for over a decade. ${ }^{3-8}$ It is recommended that a medical interventional cardiologist receive an additional year of fellowship training, and later perform at least 50 coronary angioplasty procedures annually to maintain adequate clinical skills. Given the wide variety of interventional techniques and cardiovascular lesions to which they are applied in children, one would assume that a similar minimum case volume should be required of a pediatric interventional cardiologist. Interventional cardiac catheterization in children requires a high degree of technical expertise and clinical judgment. More than any other endeavor in pediatric cardiology, interventional catheterization is associated with substantial risk to the patient. It is therefore imperative that physicians be properly trained to perform these procedures. Twelve to 18 months of fellowship training should be dedicated to acquiring cardiac catheterization and interventional skills, under the guidance of an experienced pediatric interventional cardiologist. In some instances, a fourth year of training may be required to gain such experience. Practice guidelines are more difficult to define, but are just as important to assure that physicians maintain adequate technical skills and judgment. What is the minimum number of interventional cases needed to maintain clinical competence in pediatric interventional cardiology? I recently asked this question in an informal survey of directors of several pediatric catheterization programs. The recommended minimum volume ranged from 25-75 (median 50) pediatric interventional cases annually per physician. To provide a focused ongoing experience and to assure high quality patient care, each program surveyed has designated one or two pediatric cardiologists to perform interventional cases; other physicians who perform cardiac catheterizations are restricted to diagnostic studies only.

Training and practice guidelines are vital to the future health of our patients and of our specialty. Pediatric interventional cardiologists are best qualified to define the essential elements of adequate training, and the practice guidelines necessary to assure ongoing 
competence in this highly technical field. It is imperative, therefore, that we become actively involved in the development of guidelines for our specialty. If we fail to address these issues, guidelines are likely to be developed by third parties whose primary goal may not be to secure the highest quality cardiovascular care for children.

\section{References}

1. Gray DT, Fyler DC, Walker AM, et al. Clinical outcomes and costs of transcatheter as compared with surgical closure of patent duclus arteriosus. N Engl J Med 1993;329:1517-1523.

2. Allen HD, Driscoll DJ, Fricker FJ, et al. Guidelines for pediatric therapeutic cardiac catheterization. Circulation 1991;84: $2248-2258$
3. Pepine CJ, Babb JD, Brinker JA, et al. Task Force 3: Training in cardiac catheterization and interventional cardiology. JACC $1995 ; 25: 14-16$

4. American College of Cardiology/American Heart Association Ad Hoc Task Force on Cardiac Catheterization. ACC/AHA guidelines for cardiac catheterization and cardiac cathetcrization laboratories. JACC 1991:18:1149-1 182.

5. Guidelines for professional staff privileges in the cardiac catheterization laboratory. Cathet Cardiovasc Diagn 1990;21: $203 \cdots 204$.

6. American College of Cardiology/American Heart Association Task Force on Assessment of Diagnostic and Therapeutic Cardiovascular Procedures. Guidelines for percutaneous translumi nal coronary angioplasty. Circulation 1988;78:486-502.

7. Guidelines for credentialing and facilities for performance of coronary angioplasty. Cathet Cardiovasc Diag 1988;15: $136-138$

8. William DO, Gruentzig A, Kent KM. Guidelines for the performance of percutaneous transluminal coronary angioplasty. Circulation 1982:66:693-694 